\title{
Mental Health and Service Impacts During COVID-19 for Individuals with Serious Mental Illnesses Recently Released from Prison and Jail
}

\author{
Stacey L. Barrenger, PhD \\ Lynden Bond, LMSW
}

\begin{abstract}
Individuals recently released from jail or prison with serious mental illnesses may be vulnerable to the coronavirus pandemic. This study aims to understand how they experienced the pandemic during initial stay-at-home orders in New York City. Structured surveys and in-depth semistructured interviews examined the impact of the pandemic on participants. Survey responses are presented as percentages. Thematic analysis was used to code and analyze in-depth interviews. All participants $(N=5)$ knew about the coronavirus pandemic, and most took steps to minimize risk. Participants experienced changes to their services, including suspensions of some supportive services. They also reported an increase in psychiatric symptoms but utilized a variety of coping mechanisms in response. Community reintegration was essentially on hold as supportive services were suspended. Comprehensive reentry services may need to be adapted during the pandemic to address the multiple needs of individuals and to facilitate community reintegration.
\end{abstract}

Individuals with serious mental illnesses (schizophrenia, schizoaffective disorder, bipolar I disorder, and major depression) are overrepresented within the criminal justice system ${ }^{1,2}$ and encounter many challenges, such as unstable housing, lack of income, and barriers to accessing healthcare as they return to the community from jail or prison. ${ }^{3-5}$ A connection to supportive

Stacey L. Barrenger is now at the Psychiatry Department in the College of Medicine at Northeast Ohio Medical University.

Address correspondence to Stacey L. Barrenger, PhD, Silver School of Social Work, New York University, 1 Washington Square North, New York, NY 10003, USA. Email: sbarrenger@ neomed.edu.

Lynden Bond, LMSW, Silver School of Social Work, New York University, New York, NY, USA.

Stacey L. Barrenger, PhD, Department of Psychiatry, Northeast Ohio Medical University, Rootstown, OH, USA.

Journal of Behavioral Health Services \& Research, 2021. 610-616. (c) 2021 National Council for Behavioral Health. DOI 10.1007/s11414-021-09759-z 
services, like case management, can help to improve linkages to services but does not always prevent a return to jail or prison. ${ }^{6,7}$

As COVID-19 spreads throughout the USA, jails and prisons have been greatly impacted. ${ }^{8}$ Concerns about the health and welfare of incarcerated individuals have prompted advocacy campaigns focused on decarceration. ${ }^{9}$ While this may reduce infection rates in jails and prisons, few have focused on the individuals released from these settings. Community reentry from prison is a health risk; individuals are $13 \times$ at risk for death due to homicide, suicide, cardiovascular disease, and drug overdose in the first 2 weeks post-release, ${ }^{10}$ and these risks may be heightened during the pandemic.

Individuals with serious mental illnesses recently released from jail or prison may be particularly susceptible to the effects of the coronavirus. During reentry, individuals have few resources, often lack social support, and are newly connected to mental health and other services. They may live in conditions that pose additional health risks, like homeless shelters, or may be returning to communities greatly impacted by the virus. ${ }^{11,12}$ These intersecting factors make this group especially vulnerable as they return to the community.

Many mental health and other supportive services have transitioned to telehealth during the pandemic ${ }^{13,14}$ we do not know how this new mode of care has affected those with mental illnesses released from jail or prison in the past year. While there is a growing body of research on how healthcare organizations have adapted to providing services during the pandemic, ${ }^{15,16}$ little research has focused on the perspectives of individuals and their pandemic experiences. ${ }^{17,18}$ This brief report provides a snapshot into the experiences of individuals with mental illnesses who were recently released from incarceration, during the start of the pandemic and the early days of shutdown orders in New York City. This study seeks to understand what individuals know about the virus, how they are responding to it, and if they have experienced any service changes during stay-at-home orders.

\section{Methods}

This study is part of a pilot feasibility and acceptability study examining reentry for individuals with mental illnesses living in New York City. Adults released from jail or prison in the past 6 months who reported a diagnosis of schizophrenia, schizoaffective disorder, psychotic disorder, bipolar I disorder, or major depression were recruited. Data collection included a survey at baseline, 3 months, and 6 months, and an in-depth interview after the second survey. Participants provided multiple means of contact, as research with this population often results in high attrition rates. Surveys and in-depth interviews asked about reentry experiences in addition to internal characteristics that may contribute to community tenure. At the baseline survey, trained research assistants executed informed consent by informing individuals what would be required of them over the course of the study, reviewing the risks and benefits of participating in the study, and apprising them of measures to maintain confidentiality.

In March of 2020, all in-person research activities were suspended due to the coronavirus and the New York state was under stay-at-home orders that would be extended until June 27, 2020. These two occurrences in tandem shut down recruitment to the study. At this time, 19 participants were enrolled, with 14 completing the baseline survey, seven completing the 3-month survey, and three completing the in-depth interview. In line with approved remote research procedures, the follow-up survey was converted into an electronic version with online access. Individuals had the option of self-administering the survey online or answering survey questions via phone. Both the survey and in-depth interview guides were amended to ask about individuals' knowledge about COVID-19, their concerns about contracting the virus, and the impact of the virus on their reentry experiences. All ethical standards for research involving human participants were followed. The 
original study protocol and amendments were approved by the Institutional Review Board at the university.

Despite having multiple means to contact individuals, of the 14 participants who had completed baseline interviews, nine could not be reached. Three had been re-incarcerated and six could not be located. Five individuals completed the amended 6-month survey and the in-depth interview. This analysis draws from these data, focusing on participants' experiences during the pandemic. Survey responses are presented as percentages and in-depth interviews were coded and analyzed utilizing thematic analysis techniques. ${ }^{19}$ While this sample cannot be representative of all individuals recently released from jail or prison, it provides a snapshot of how the pandemic and resulting social distancing measures have impacted vulnerable individuals.

\section{Results}

All participants $(N=5)$ reported having heard "a great deal" about coronavirus and COVID-19 and all but one was very concerned about contracting the virus. A variety of behavior changes, from those who expressed concern $(80 \%, n=4)$, were endorsed including staying indoors and away from public places, canceling plans involving other people, and avoiding public transportation. Despite their concerns, all participants reported using mental health and other supportive services in the last 30 days, like housing, legal, substance use, case management, and medical services. All participants $(100 \%, n=5)$ also reported changes in service provision under stay-at-home orders, including changes in program hours, staffing, and service delivery, with some programs shutting down entirely. These changes led to difficulties in accessing care or services in the past month.

Analysis of the in-depth interviews produced three main themes: managing risk, coping with the pandemic, and navigating reentry. All participants spoke of managing risk during stay-at-home orders. Participants were well-informed about the coronavirus and reported engaging in social distancing, wearing masks, and increasing hygiene practices, but they also identified having to experience unavoidable risks due to accessing needed behavioral health and other supportive services. For example, two participants were enrolled in an outpatient medication-assisted treatment (MAT) program, which required them to go to the agency to receive medication. Miguel (all names are pseudonyms), weighing the risk of not receiving his medication against contracting the virus, stated, "So, I have no choice but to deal with the not social distancing, because they stick us on an elevator," while Fred managed his risk by taking cabs to avoid public transportation saying, "I've been wearing a mask. I don't go out. I don't run around the streets like I used to....I go to my program. I get medicated. I get in a cab, I come home and I'm home all day. Every single day. I'm very rarely out in the streets because I'm too scared to be in the streets at this time." Most faced risks in their housing and living situations. One participant lived with his brother, who had been exposed to the virus at work and had to quarantine in the apartment for 14 days. Three participants lived in congregate settings (shelters or supportive housing), which led to increased risk of being exposed. Jason, who lived in supportive housing and had a compromised immune system, was sensitive to the lack of control over others' actions stating:

\footnotetext{
I have to share the bathroom, share the kitchen, share the - the area where I wash my dishes, so it's not like leaving my home jeopardizes my circumstances, leaving my room jeopardizes my circumstances...because every time I leave my - my room, which has no sink or no toilet or no kitchen or cleaning facilities, I'm putting myself in danger, because I'm opening up my physical health and my hygiene to people who are not living at the same level of clarity as I am about the pandemic.
}

Kevin, who was residing in a congregate shelter, described the ways in which the program attempted to manage the risk among residents, saying, "You know, gotta be six feet apart. Uh, you know, you gotta maintain social distancing and you know, you gotta wear the mask." But Dennis, 
who stayed at a different shelter, highlighted the lack of hygiene resources at the shelter as a cause for concern, "Um, you know, people don't bathe. Um, the shelter, they don't have a - uh, basic necessities, there are no towels, anything, there's no soap." Along with the everyday risks, like grocery shopping, that everyone faces, our participants also managed risks in their housing and in accessing needed health services.

Participants identified two areas that were greatly impacted by stay-at-home orders: service changes and their mental health but developed several ways of coping with the pandemic. While no one reported an interruption in psychiatric medications, participants identified an increase in anxious and depressive symptoms due to the pandemic. Fred described the impact on his mental health, saying, "it's caused me to be nervous, to be anxious.... get agitated quicker." Miguel referred to the news of deaths due to the virus as contributing to his increase in symptoms:

It's [the pandemic] impacted my mental health in a very negative way because I already deal with anxiety disorder. So I have a manic depression, I have schizophrenia. I have post-traumatic stress disorder....it even brings back, um, traumatic memories....of people dying....in my past. So it's been really, really difficult to cope with, um, with what life is now.

While many providers switched to telehealth services, some programs closed completely. Notably, Jason could not access healthcare services, explaining, "I have a primary care physician and a dentist and all these people at a health organization...but they're closed. So, they're not available to me." The participants living in shelters or congregate housing reported that applications for permanent housing had stalled leaving them in risky conditions. Kevin stated that, "You know, um, [there's] help out there but you know, it's put on hold because everything is on hold right now.... The process [of applying for permanent housing] can still get done but it's like a long process." Dennis had a similar experience, saying, "Like I said, my housing that was put on hold because, um, everybody's staying at home... and, uh, that affected me, yes, with my housing because nobody's going to work, nobody wants to do anything."

For most participants, telehealth services did not work for them as they did not have the adequate resources (phone or internet access) to engage with providers. Even with needed technology, some were not familiar with how to operate the technology due to years of incarceration. This was true for Dennis, who described, "They wanna do [counseling] over the phone, or... a video, like Zoom, and....I'm not tech savvy and I try to get on Zoom a couple times and just had a lot of difficulty and I never pursued it." Unable to access counseling services, he felt it was up to him to manage his increasing symptoms, "I have to bottle it up. You know, and keep it in because I have really nobody to confide in." This sentiment was shared by others, notably Jason who shared, "I can't interact with any of my caregivers or doctors, I don't feel safe taking public transportation, and travelling out of my neighborhood to places of interest that I enjoy." Some participants felt that this inability to access services was like when they were incarcerated and needed to mostly rely on themselves to manage in a difficult situation. In fact, Jason described himself as being "like the Energizer Bunny, in that I can navigate any situation that you put in front of me". Miguel related a myriad of ways he was dealing with his anxiety, "I try to stay busy in the house. I'm always cooking and I'm on home projects...I try to exercise....[I take] my medication, and watch the TV." Other participants reported they expressed gratitude, tried deep breathing, or exercised to deal with the increased anxiety due to the pandemic.

Participants related the many ways they were navigating reentry, but most said that isolating due to the pandemic reminded them of being incarcerated. They described both the physical and social isolation as similar to incarceration, but also recognized that even under quarantine, they had more freedoms as Jason related, “ ... relative to what I personally have just experienced, the - the coronavirus and the quarantine and the fear and the people dying, sort of fades by comparison to being... locked up, being in handcuffs...so it's sort of, like, all relative to me." Miguel felt the 
pandemic impacted his community reentry as closures and social distancing measures prevented him from integrating back into society in a meaningful way:

Well, I just came out of jail not too long ago and then, um, trying to assimilate back into, um, society. And then, um, a few weeks later all this [the pandemic] happened and everything closed down...I don't, I haven't felt part of society. Um, so I've been trying to get back into society... it's like I can't because of what's going on. Oh, I still feel like I'm not a part of anything, a part of the community or anything.

Finally, Fred recognized the irony of finally being out of prison but facing possible sickness or death from the virus stating, "I'm finally crime free. I'm not breaking the law anymore.....and I wanna live, even though I'm broke but that's okay. You know, I'm happy just to be home and free. But now that this is happening, it's putting fear in my heart... who knows if anyone's gonna make it through this?"

\section{Discussion}

The findings show how the pandemic impacted reentry experiences of those with mental illnesses as services and supports were curtailed, especially around securing permanent housing. Individuals experienced an increase in depressive and anxious symptoms, felt socially isolated from others, and expressed that their community reintegration was put on hold because of stay-athome orders. Participants expressed concern about contracting the virus; yet, either due to living situations, the need for treatment, or in one case, having a job, all needed to enter public spaces and manage their risk exposure. Despite these shared experiences, one size does not fit all, as our participants had different experiences with accessing or attending services. Notably, some were able to accommodate the services changes due to the pandemic, but for some these changes resulted in not receiving sufficient services or not receiving needed services at all.

All participants reported an increase in mental health symptoms due to social isolation and concern about contracting the virus, supporting recent findings among a similar population. ${ }^{17}$ While the social isolation was difficult, participants managed these symptoms through strategies developed during incarceration. They employed numerous self-care techniques (expressing gratitude, deep breathing, exercising, cooking, and watching TV) to cope, indicating they were accustomed to managing on their own as they often needed to do while incarcerated.

Due to the small sample size, these findings are not representative of all individuals recently released from jail or prison or those released during the pandemic. The participants were connected to services prior to stay-at-home orders and the corresponding transition to telehealth services. It is unclear how individuals released during the pandemic are receiving services when according to a national survey, $75 \%$ of reentry programs have curtailed or canceled services. ${ }^{20}$ While New York City took immediate steps to provide comprehensive reentry services to individuals released from jail during the pandemic, these efforts were met with mixed reports from the City and service providers. In March 2020, the City implemented the 6-A Early Release Program, providing intensive intervention in lieu of incarceration, and touted the responsiveness of case workers to assist in providing critical reentry services such as helping individuals obtain cell phones and housing. ${ }^{21}$ However, service providers have reported that the City's reentry programs have failed to provide enough support to individuals being released from jail during the pandemic and lacked communication and coordination between agencies, leading to gaps in critical services such as housing and medical care. ${ }^{22}$ While the findings may not be generalizable, they provide some insight into how some individuals released from incarceration experienced the early months of the pandemic. The views expressed may not represent a subset of our population as six participants could not be contacted. It is possible that their experiences are different from the ones represented in these findings. Finally, even under the best conditions, longitudinal research with this population remains difficult as the transient nature of reentry coupled with minimal resources contributes to 
high rates of attrition. Even with multiple means of contact, the attrition rates were high for this study. Utilizing novel methods such as a study website or Facebook page may provide additional means for those with access to technology to stay in contact with the study. Utilizing peer researchers to perform outreaches to study participants may be another avenue to reduce study attrition among this vulnerable and transient population.

Despite these limitations, there are some important findings from the small sample that are pertinent under the current context. Although individuals missed out on needed services, they showed remarkable resiliency in managing symptoms and navigating risky situations. However, it is possible that this subgroup may be more resilient than the group who could not be located. It is quite possible that those who could not be located were facing more challenges during this time which prevented them from continued study participation. In planning for services during the pandemic, providers should not forget to help individuals draw from personal strengths to manage increases in symptoms and fears. This may be particularly true for those who learned to manage psychiatric symptoms under risky situations, like incarceration, with limited access to mental health services. In fact, organizations should draw from a continuum of services and service delivery, ${ }^{23}$ including natural supports to meet the multiple needs of vulnerable populations.

\section{Implications for Behavioral Health}

As the nation is completing the first year of the pandemic, this vulnerable population must not be overlooked. While telehealth services have bridged the gap between stay-at-home orders and service providers, ${ }^{14}$ this service mode does not work for those with no access to telephone or internet services ${ }^{12}$ and may be challenging for those who have not mastered technology due to being incarcerated. Additionally, telehealth services are not always an appropriate form of care, particularly for marginalized individuals utilizing medication-assisted treatment for substance use disorders, or for medical-related treatment requiring physical evaluations or procedures, leading to gaps in accessing critical healthcare. As such, many of our participants were held in a limbo state as providers focused mainly on providing psychiatric and counseling services while other supportive and medical services were stopped indefinitely. As the event horizon for the pandemic has lengthened, some communities have adapted by responding to the reentry needs of individuals with serious mental illnesses. Yet, there continue to be challenges to implementation during this public health crisis. Future research with this population must include an examination of the past and continued impact of COVID-19 on their community transitions and community tenure.

With increased knowledge of the transmission and risk factors associated with the coronavirus, comprehensive behavioral health and reentry services should be continuously adapted to address the multiple needs of this vulnerable population. For example, programs can consider using hybridlike service delivery models, using both in-person and telehealth-based services which minimize risks while maximizing service benefits and sufficiently meet individual needs. ${ }^{23}$ In addition, providers must recognize the continued economic, social, and physical impacts of the pandemic and, when appropriate, focus on addressing basic needs such as housing, food security, and benefits assistance in addition to behavioral health needs. ${ }^{23}$ As has been observed with ethnic and minority populations, the pandemic has exposed health disparities that are only heightened under the current conditions. These health disparities also include those with serious mental illnesses. In addition to ensuring that individuals receive behavioral health, health, and social services during community reentry, efforts need to focus on ensuring this population is not overlooked for continued COVID-19 testing and vaccinations.

Funding This research was funded by the Silver School of Social Work Seed Grant, New York University. 


\section{Declarations}

Conflict of interest The authors declare no competing interests.

\section{References}

1. Bronson J, Berzofsky, M. Indicators of mental health problems reported by prisoners and jail inmates, 2011-12 2017. NCJ 250612. Washington, D.C. U.S. Department of Justice, Bureau of Justice Statistics, 2017.

2. Steadman HJ, Osher FC, Robbins PC, et al. Prevalence of serious mental illness among jail inmates. Psychiatric Services. 2009;6:761765 .

3. Barrenger SL, Draine, J. You don't get no help: the role of community context in effectiveness of evidence-based treatments for people with mental illnesses leaving prison. American Journal of Psychiatric Rehabilitation. 2013;1:154-178.

4. Barrenger S, Draine J, Angell B, et al. Reincarceration risk among men with mental illnesses leaving prison: A risk environment analysis. Community Mental Health Journal. 2017;53:883-892.

5. Blank Wilson, A. How people with serious mental illness seek help after leaving jail. Qualitative Health Research. 2013;23(12):15751590 .

6. Morgan RD, Flora DB, Kroner DG, et al. Treating offenders with mental illness: A research synthesis. Law and Human Behavior. 2011;36(1):37-50.

7. Skeem JL, Manchak S, Peterson JK. Correctional policy for offenders with mental illness: Creating a new paradigm for recidivism reduction. Law and Human Behavior. 2011;35:110-126.

8. Barnert E, Ahalt C, Williams B. Prisons: Amplifers of the COVID-19 pandemic hiding in plain sight. American Journal of Public Health. 2020;110(7):964-966.

9. Nowotny K, Bailey Z, Omori M, et al. COVID-19 exposes need for progressive criminal justice reform. American Journal of Public Health. 2020;110(7):967-968.

10. Binswanger I, Stern MF, Deyo RA, et al. Release from prison-a high risk of death for former inmates. New England Journal of Medicine. 2017;356(2):157-165.

11. Coughlin S, Moore JX, George V, et al. COVID-19 among African Americans: From preliminary epidemiological surveillance data to public health action. American Journal of Public Health. 2020;110(8):1157-1158.

12. Sullivan S, Bell K, Spears A, et al. No community left behind: A call for action during the COVID-19 pandemic. Psychiatric Services. 2020;72(1):89-90.

13. Pinals DA, Hepburn B, Parks J, et al. The behavioral health system and its response to COVID-19: A snapshot perspective. Psychiatric Services. 2020;71(10):1070-1074.

14. Yellowless P, Nakagawa K, Pakyurek M, et al. Rapid conversion of an outpatient psychiatric clinic to a $100 \%$ virtual telepsychiatry clinic in response to COVID-19. Psychiatric Services. 2020;71(7):749-752.

15. Guan I, Kirwan N, Beder M, et al. Adaptations and innovations to minimize service disruption for patients with severe mental illness during COVID-19: Perspective and reflections from an assertive community psychiatry program. Community Mental Health Journal. 2021;57:10-17.

16. Murphy AA, Karyczak S, Dolce JN, et al. Challenges experienced by behavioral health organizations in New York resulting from COVID-19: A qualitative analysis. Community Mental Health Journal. 2020;57:111-120.

17. Costa M, Pavlo A, Reis G., et al. COVID19 concerns among persons with mental illness. Psychiatric Services. 2020;71(11):1188-1190.

18. Florence AC, Miller R, Bellamy C, et al. When reality breaks from us: Lived experience wisdom in the Covid-19 era. Psychosis. 2020;12(4):363-367.

19. Braun V. \& Clark V. Using thematic analysis in psychology. Qualitative Research in Psychology. 2006;3:77-101.

20. Council of State Governments Justice Center. Survey shows reentry services halting across U.S. Available online at https:// csgjusticecenter.org/survey-shows-reentry-services-halting-across-u-s/. Accessed May 25, 2020.

21. Center for Court Innovation. Rikers 6-A early release program results after one month of operations [briefing note]. Available online at https://www.nycja.org/assets/Rikers-Early-Release-Briefing-Note-4.30.2020_-FINAL.pdf. Accessed March 1, 2021.

22. Karthikeyan, D. De Blasio administration fails to provide proper reentry services for those leaving jail amid pandemic, providers say. Gotham Gazette. Available online at https:/www.gothamgazette.com/city/9800-de-blasio-administration-failing-provide-proper-reentry-services-leaving-rikers-jail-amid-coronavirus. Accessed March 1, 2021.

23. Kopelovich SL, Monroe-DeVita M, Buck BE., et al. Community mental health care delivery during the COVID-19 pandemic: Practical strategies for improving care for people with serious mental illness. Community Mental Health Journal. 2020;57:405-415

Publisher's Note Springer Nature remains neutral with regard to jurisdictional claims in published maps and institutional affiliations. 